\title{
Sexuality, Disability, and Reproductive Issues Through the Lifespan
}

\author{
Sandra S. Cole ${ }^{1.3}$ and Theodore M. Cole ${ }^{2}$
}

KEY WORDS: Reproduction; disability; sexuality.

The decade of the 1990s presents an opportunity for America to reflect on the progress that has been made in services, attitudes, and opportunities for people with disabilities. In her book, Sex, Society and the Disabled, Robinault (1978) reminds us that Margaret Mead said, "The character of a culture is judged by the way it treats its disabled." In the United States we are now implementing the Americans with Disabilities Act (ADA), and Margaret Mead would be pleased to judge our culture by our commitment to the ADA.

Biology is the determinant of anatomic sex. It is not the determinant of behavioral sex, and the primate learns sexual behavior. More is needed than biology alone-education influences the final product of sexual health. However, in animals, sexual behavior is determined neurochemically. It is related to reproduction, which is its final intent. In humans, reproduction and sexual behavior are not synonymous. Men and women must learn not only about sexuality, but they must also learn about sexual relationships. Add to this the need to learn about love, and it becomes apparent that both the tasks and the rewards become complex. Beyond sexual love is the more generic love for humankind, a noncoital love involving personal interaction and charm.

Historically, disability in the Western world has a grisly past. In early

'Professor, Department of Physical Medicine and Rehabilitation, University of Michigan Hospitals, Director, Sexuality Training Center and Sexuality Evaluation Clinic, University of Michigan Hospitals, Ann Arbor, Michigan.

${ }^{2}$ Professor and Chairman, Department of Physical Medicine and Rehabilitation, University of Michigan Hospitals, Ann Arbor, Michigan.

${ }^{3}$ Address correspondence to Department of Physical Medicine and Rehabilitation, University of Michigan Hospitals, 1500 East Medical Center Drive, Ann Arbor, Michigan, 48109.

Excerpted from Reproductive Issues for Persons with Physical Disabilities, Brookes Publishing Company. Reprinted with permission of the publisher. 
cultures, an imperfect child was usually killed. In the Middle Ages, such a child was tolerated but was the object of ridicule or fear. Court jesters were sometimes the adult products of birth defects. By the time of the Renaissance, many people with disabilities could look forward to living out their adult lives in asylums.

It was not until the 18th century that the first "modern care" for people with disabilities was begun, in Switzerland. In the 19th century, the first glimmers of education and scientific inquiry into physical disabilities and mental retardation were seen.

By the middle of the 20th century, what has come to be known as modern rehabilitation first appeared. It was the by-product of a society sympathetic toward survivors of military encounters. Now at the close of the 20th century, we are just beginning to demonstrate our concerns for personhood, body image, and human sexuality as a part of rehabilitation (Robinault, 1978).

\section{AN OVERVIEW}

Sexuality and reproductive issues encountered by people with physical disabilities during early childhood, adolescence, and early reproductive years are likely to be different from those that people without disabilities experience. Earlier years (infancy) and later years (adult reproductive years) have many similarities for both groups.

It is most convenient to think about these issues from four points of view: 1) sexuality and developmental disabilities, 2) sexuality and acquired disabilities, 3) reproductive issues, and 4) issues pertaining to the aging process.

Early onset disabilities are likely to affect sexual development in terms of gender roles, the language of sex, privacy, self-exploration, sex education, and personal learning. Socialization experiences in early childhood give direction and meaning to adult sexuality. Examples of medical correlates include musculoskeletal disabilities and neurogenic impairments that can affect fertility and sexual options (Hanks \& Poplin, 1981).

Disabilities that are acquired after sexual maturity may affect these same areas, but in different ways. Gender roles may become blurred, past sexual patterns of activity may impede creativity needed after disability, and medical experiences may have a desexualizing effect upon self-esteem and libido. Birth control, sexually transmitted diseases, pregnancy, reproduction, and parenting present similar concerns for early onset and adult onset disabilities.

Individuals with and without disabilities have the same rights to information, services, and to health service providers with adequate knowledge, sensitivity, and experience in areas of sexual development. Self-empowerment, life skills, parenting, and medical concerns are common to everyone, but these are 
issues that may need special attention in individuals with physical disabilities. As individuals mature, new problems may be added to underlying disability issues: for example, further confusion of gender role, stigma, need for more adaptive equipment, societal expectations of the aging adult, and the anticipated transition from health to illness (Frank, 1988; Goffman, 1963).

\section{DEVELOPMENTAL ISSUES}

\section{Gender}

As children grow, a tremendous focus of energy and instruction, both from the individual and society, is on the careful preparation and development of the gender role of female or male. Much of the childhood experience lays the foundation for the masculinity and femininity of personalities and relationships with others. It must be recognized that the presence of a physical disability influences and in some ways may limit the development of psychosexual/ social maturity.

It is reasonable to assume that parents, in anticipation of the birth of a child, have fantasies and dreams of what their "perfect little child" will be as a man or a woman. Needless to say, parents are generally not prepared for the birth of a child with a disability, and, although they can learn to understand and work with the presence of a disability, many of their dreams, plans, and preparations for the child and for the family will be altered (Darling, 1988; Shapiro, 1983).

\section{Privacy}

In the early years when children first demonstrate independence and curiosity, including curiosity about themselves and their sexual bodies, the child with a disability probably experiences some alteration in natural opportunities for privacy. He or she may be more closely observed or supervised by family or caregivers and therefore less able to be spontaneous and private with sexual curiosity than a typically developing child. This lack of privacy can affect a child's perception of his or her body, its function, and personal boundaries regarding appropriate or inappropriate touch. Most learning in children is done spontaneously and includes a great deal of physical involvement with other children, adults, and with the environment-physical involvement being a catalyst to learning. Spontaneity is dramatically affected and can be severely limited by the presence of a disability (e.g., affecting vision, mobility, speech and/ or hearing) (Cole \& Cole, 1990). 


\section{Language and Communication Skills}

During these years, children are simultaneously gaining language skills: first, recognizing language; second, gaining cognition; and third, developing individual skills to communicate with parents, family, and the environment. During these early years, children are also obtaining the "language of sex," learning terms and phrases for body parts and behaviors, and striving to gain an understanding of word usage in expressing thoughts and feelings about sex and sexuality. Their efforts sometimes range from embarrassing and silly to dramatic. This phase is a time for adults and parents to provide children with accurate language for body parts and their functions, appropriate and accurate sex education, in addition to the usual private and personal words, names, and sexual expressions used within the family.

It is helpful for all children, including the child with a disability, to know correct words, their usage, and to be able to express him- or herself appropriately regarding sexual matters. This knowledge and ability increases the child's self-confidence and self-esteem and can be a contributing factor in prevention of sexual abuse, molestation, or exploitation. Increased knowledge, awareness, and language confidence can help the child to recognize inappropriate behavior, abuse, or violence that may be imposed on him or her, since persons with disabilities are particularly vulnerable to sexual perpetrators (Cole, 1986; Sobsey \& Varnhagen, 1988).

Many common expletives in adult language make reference to sexual activities. A child very likely will have heard such "swearing" and, most naturally, tries to repeat those words to emulate adult behavior, even if the words are not understood. This spontaneous behavior of children frequently generates a startled reaction from adults and can sometimes result in an immediate opportunity to provide sex education, helping the child to distinguish appropriate and inappropriate language and social situations. (Unfortunately, occasionally the response from an adult is to admonish and punish the child.) A child with a disability may be quite insulated from having these spontaneous experiences. For children with disabilities, particularly those who have limited mobility, it is entirely possible that a part of their development may be influenced by isolation or limited access to peers, and that they may not have had many opportunities to explore and learn about life and its events with their peers. As children with disabilities get older, these life experience omissions become more evident and they may experience social handicaps because of their lack of awareness or understanding of society and the behaviors of the adult world. Since most information about sex is learned quietly, covertly, and is greatly and dramatically influenced by peers and the media, children with disabilities may experience distinct limitations in knowledge and communication about sex education and sexual behavior (Robinault, 1978). 


\section{Touch}

Touch is a major element in human development and has a powerful effect on all individuals. Infants and children first learn feelings of security, intimacy, and bonding through touch. Love and affection are expressed through touch and language. Children with physical disabilities have additional touch experiences that relate to medical care procedures or to assistance with activities of daily living (ADL).

Children with physical disabilities may require specific assistance with movement or positioning and may require apparatus or equipment to facilitate ambulation and mobility. As a result, they may experience a profound difference between being handled for management and health care purposes and being touched and held for tender and loving purposes. Many children with disabilities experience far more "handling" then tender loving caresses. Similarly, having a disability that requires medical attention presents the possibility of more public nudity than that experienced by a typical child. Having one's body examined and impersonally handled by health care providers often involves this type of physical exposure, and this type of touch most often is not negotiated with the child by health care providers. It is a common experience for individuals with disabilities to have medical procedures, examinations, and inquiries made by health care providers who touch their bodies for purposes of examination but who neglect to be sensitive to this intrusion. Any effort to acknowledge and negotiate personal boundaries of touch is generally overlooked, and the implications of being touched by strangers usually are left unresolved. Obviously, vulnerability to exploitation, misinterpretation, or misinformation is increased with these situations.

Such behavior is common, although inappropriate, in the health care professions. For example, a child with an orthopedic condition may have experienced many examinations that take place with five or more members of a health care team in attendance. In addition, the procedures may be videotaped and the child may be wearing only underpants during the examination. Vulnerability during pubescent years when children are naturally modest, particularly as they experience genital development, is heightened. Perhaps one of the most important lessons for professionals in health care to learn is to respect the individuality, personal dignity, and privacy of patients, something that is not often demonstrated in public examinations in amphitheaters or lecture halls.

When boundaries of appropriate touch are not clear, a child with a disability may not learn to distinguish between appropriate and inappropriate touch and may not have a healthy understanding of the subtleties between public and private nudity and public and private sexual behavior. For some children, especially in institutions, inappropriate touch may have been the only form of affection they have received, and, therefore, their ability to distinguish appropriate- 
ness has been impaired. It is well known that health care providers are not infrequently the abusers of persons with disabilities (Griffith, Cole, \& Cole, 1990; Sobsey \& Vamhagen, 1988).

These issues add to the well-established societal fact that adults handle and interact differently with male and female children (Allgeier \& Allgeier, 1991). Adults tend to be more aggressive and physically firm in the way boys are handled in daily activities. Girls are more frequently stroked and caressed, spoken to softly, and handled gently. With all children, society begins to shape the gender roles of masculine and feminine from infancy. A child with a disability may experience additional limitations by being unable to participate fully in his or her expected and implied gender role (Gordon, 1975).

\section{Self-Exploration}

In early childhood, self-exploration of genitals is a natural activity. Male genitals are exposed, available, conspicuous, and of great interest to the little boy. Female genitals are hidden, less conspicuous, and much more mysterious and difficult to access for the little girl. For boys who are easily able to handle their own genitals, there is a direct opportunity to touch and stimulate their penises. Girls experience a more diffuse and sensual kind of touch in sexual expression, through caressing and stroking, as their genitals are hidden and more obscure. These distinguishing sexual aspects of masculinity and femininity are carried into adult sexual roles, each role having been developed and refined through puberty and adolescence. Some children with disabilities may have difficulty reaching and touching their own genitals. They also may lack the ability to experience touch sensation in their genitals. They may not directly experience their whole bodies, particularly if there is sensory loss and no feeling in some parts of their bodies. The spontaneous, curious, and innocent ways in which children learn about their bodies through touch may be less prevalent in the child with a disability than in typical peers, either through personal sensory limitations or through limitations such as lack of privacy, the need for assistance, and being discouraged in these activities by adults or by an environment controlled by adults (i.e., an institution).

The situations that have been described indicate the specific need for accurate and comprehensive sex education and gender role preparation for children with disabilities. Society must be more diligent in providing opportunities for children with early onset disabilities to acquire knowledge about themselves as sexual individuals, about sexual mores in the culture, and about the informal world of sex that their nondisabled peers more easily access. Toward that end, parents and educational systems are encouraged to provide structured sex education and to help children with disabilities to obtain accurate information about sexual health and to form positive role models for themselves. 


\section{Adolescence}

During adolescence, the most common means of learning about sex is through private, informal, and perhaps secret opportunities alone or with others to rehearse the roles of a sexual man or woman. The need for opportunities for sexual rehearsal cannot be overemphasized, as it is during these occasions that adolescents begin to sort out the many mixed messages they have learned about sex and sexuality (Money, 1986). There are dramatic changes in their bodies as secondary sex characteristics begin to develop. It is a time for practicing and refining one's masculinity or femininity, and, similarly, it is during this time that the young adolescent boy and girl will begin to talk openly with peers about matters of sexual development, behavior, and desires. They will practice relating to peers and to the opposite gender in sexual ways and will enter into the dramatic, sensuous, and momentous experiences of rehearsing their adult roles (Gordon \& Gordon, 1983).

As many adults recall, some of the most significant memories of adolescent sexual development were of those events that occurred privately and unobserved. These opportunities of spontaneous learning may not be available to adolescents with disabilities, who often lack easy accessibility to other adolescents. Dynamics change considerably if, as a teen, one has to be catheterized, transferred into an electric wheelchair, assisted into a van, and delivered to a friend's home or public event where further assistance is needed to get into the building. At the end of the event, all of the maintenance activities are repeated in reverse. Spontaneity and social experiences and risk-taking are inhibited in these circumstances. Women who experience a disability before adolescence report their first social and sexual experiences later than their peers for these reasons (Rousso, 1988).

Society has a tendency to reinforce the dependency of children and young adults with disabilities. They are frequently infantilized in their relationships and communications with adults. A child may quickly learn that he or she is perceived and treated as helpless or less able, and this may actually facilitate the development of a "helpless individual" or "learned helplessness" (Romeis, 1983).

The pubescent child with a disability can be at risk for stigmatization by an insensitive, uncaring, and ignorant able-bodied society. At a time when conspicuousness and vulnerability is at its peak in boys and girls, the presence of a disability can further compound the difficulty of natural sexual and social development. The young person may have had so little opportunity to spontaneously interact with peers that he or she may not have the ability to develop the social skills of adolescence so necessary to carry out the culturally expected roles of male and female. 


\section{Perfection/Body Image}

Society places enormous positive value and emphasis on having a perfect body. This message is taught and reinforced in the early years by the presence of role models with perfect bodies and by the lack of role models with disabilities. Advertising and media rarely show a person with a disability. By the time a child acquires language, he or she has already learned that to be disabled is to be different, imperfect, and perhaps unacceptable (Fine \& Asch, 1985). To be imperfect is to be asexual and anonymous or overlooked in the sexual spectrum of adult life. The continuing emphasis on the healthy and physically fit adult (who must also achieve a perfect body) is a concrete message learned repeatedly from early childhood through media and advertising. This is often a further assault on the vulnerability of self-esteem for all people without perfect bodies, increasingly reinforced for persons with disabilities (Bogdan, Biklen, Shapiro, \& Spelkoman, 1982; Brolley \& Anderson, 1986; Ruffner, 1984).

\section{Social Opportunities}

Social opportunities for adolescents to interact with peers assist young adults to gain self-esteem. Clothing and fashion, music and the media, community activities, social events, and school experiences are major contributors to gender development at this age. The technical aspects of having physical access to settings where social events occur are often taken for granted by the nondisabled population. These years are times for intense peer interaction, communication, and learning by watching, doing, and rehearsing. Parental influence and values are challenged during these years and often are replaced by peer and media influence. Each adolescent matures at a different rate, but, all in all, these years are extremely full of sexual and sensual overtones, messages, and activities for those who can access them.

The sexual messages in the media about perfection and the artificiality of adult life as presented on television and in magazines tend to be one-dimensional and value-laden and provide few opportunities for the adolescent to discuss and clarify such values and their implications. These aspects of sexual socialization strongly shape and influence the emerging adult (Blackwell-Stratton, Breslin, Mayerson, \& Bailey, 1988). It is entirely possible that the adolescent with a disability may have to be a spectator on the sidelines rather than a participant in exciting and demanding experiences of social-sexual development.

Socialization opportunities are essential to growth and development. If the process is healthy, essential self-esteem is achieved through daily activities, social events, and by wearing particular clothing and observing contemporary 
fashions. It is assumed that the child has access to settings in which these events occur. For most children, the setting does not limit them to the role of spectator but allows active participation and peer interaction. The child with a physical disability may have a more passive than active role, and learning may result more from reading and watching than from doing and experiencing.

\section{Medical Issues}

More and more, medical care includes educating the individual and the family about physical, emotional, and behavioral aspects of the disability. Even so, education is frequently presented from the perspective of the health practitioner and may be more technical than useful to the individual and family (Biklen, 1988).

Issues that should be included in patient education are mobility limitations, weakness and fatigue, management of spasticity, and adaptation to somatosensory and perceptual dysfunctions (e.g., loss of vision, hearing, smell, or touch). This material is always best presented in a nonjudgmental fashion, while the educator helps to broaden the individual's and family's concept of human diversity. (Cole \& Cole, 1990).

In terms of sexuality, it is advisable to avoid a narrowly defined concept of sexuality (e.g., coitus and reproductive physiology). It is useful to include information that helps the adolescent or adult prepare for sexual fantasies and activities. Avoiding unwanted pregnancy and sexually transmitted diseases would be high on the list of desirable topics to be taught. It is all too possible that an individual with a physical disability may have alterations of body functions that interfere with the timely and accurate recognition of symptoms of sexually transmitted diseases.

Inherent in this more modern approach to health care is the assumption that the physician and other health professionals are educated and knowledgeable, both about human sexuality and the individual and the specific disability (Sandowski, 1989). It is pleasing to see that some medical examination rooms incorporate examining techniques and equipment that are user-friendly to the "disabled customer." The paraplegic woman will readily attest to the awkward, if not embarrassing, experience of being subjected to a pelvic examination that is carried out on a traditional and inaccessible examining table - too high for transfers, too narrow for safety, and with stirrups that require leg positions that may be impossible. (Ferreyra \& Hughes, 1982).

\section{ISSUES CONCERNING ACQUIRED DISABILITIES}

Disabilities acquired in early adulthood after maturation may most specifically affect the established and well-developed sense of masculine or feminine 
identity of the individual. In fact, the elaborate preparations for masculine and feminine roles of individuals are interrupted by the onset of the disability. Life plans, goals, dreams, and ideals are significantly challenged and perhaps changed by the onset of disability. The individual will most likely experience grieving, not only for specific body changes and losses, but also regarding the trauma of having one's private dreams altered, including one's sexual identification as a man or woman.

\section{Stable and Progressive Disabilities}

A disability acquired as an adult may be characterized as stable (e.g., a spinal cord injury) or progressive (e.g., multiple sclerosis). There are differences between these types of disabilities. Although the trauma of an acquired disability is undeniable, once the disability is stabilized, an individual can proceed with organizing his or her life and achieving some semblance of control, with adaptation.

It is more complicated, however, to experience a progressive disability, because it is hard to predict the course medically and physically. It is therefore difficult to be confident about being in charge or in control of daily living, spontaneity, and planning for the future. When individuals are in the stages of dating or planning a family, these uncertainties may complicate the usual challenges of relationships and family living.

Through the rich use of memory and the creative use of fantasy, much pleasure can be retained from previous sexual experiences of individuals who have acquired disabilities in adult life. Sensuality can be enhanced through memory and retained throughout various sexual pleasures and activities, even with physical changes or limitations. With some disabilities, intimate behaviors and preferred sexual positions may have to be changed or adapted. However, severe physical losses (e.g., for a male with spinal cord injury) often cause distinct and serious concerns about fertility and the ability to be a parent, in addition to general and obvious concerns about erectile dysfunction and being accepted by society as a "real" man or woman (Mooney, Cole, \& Chilgren, 1975; Neistadt \& Freda, 1987; Rabin, 1980; Sha'ked, 1981).

Partners of persons with disabilities may experience the dual roles of personal care assistants and of intimate partners. These challenges can create feelings of conflict for both the partner and the person with the disability and may contribute to stress in the relationship. Efforts must be taken by the health care worker to be sensitive to these situational dilemmas and to assist in creative problem-solving recommendations (Griffith et al., 1990).

Persons socialized in contemporary American culture are surrounded by traditional societal messages about parenting and disability (Hahn, 1981). It is imperative that health care practitioners avoid falling into the trap of stereotypical responses of pity, avoidance, infantilization, and excessive attention to per- 
sons with disabilities. Independence, personal esteem, positive body image, and positive sex messages should be emphasized. Silence from the medical community concerning disability, sexuality, and reproductive issues relays the stronger message of rejection and repression and gives the impression that parenting is not to be considered. This approach is not helpful.

\section{GENERAL MEDICAL CARE}

Men and women with acquired disabilities frequently avoid general medical care. The reason commonly given is that they believe that physicians providing general health care are not sensitive to the specific urologic and gynecologic needs and conditions of a person with a disability. Women who have disabilities often find that their gynecologist is not trained in alternative methods of conducting a pelvic examination on women with a variety of physical limitations. Many health care facilities and offices are not even accessible for the individual with a disability.

Among the general population, it is well established that sexual exploitation occurs in one out of three females and one out of seven males before they reach adulthood (Finklehor, 1979). The statistics are even more grave among those with disabilities (Mullan \& Cole, 1991; Sobsey \& Varnhagen, 1988). Early research has indicated that men and women with disabilities are more frequently sexually exploited than nondisabled persons and that it is almost always done by someone known to the individual. As is the case with all sexual exploitation, the psychological effect can be traumatic, and, when the trauma experienced is compounded by a disability and a lack of sex education or healthy sexual experiences, we can only assume that understanding and concerns about sexuality and confidence about one's sexual self-esteem are dramatically affected.

Certainly, there is great recognition that particular medical attention should be paid to persons with disabilities, not only to be observant to clues or indications of sexual abuse, but also for opportunities to provide information about birth control and the prevention of sexually transmitted diseases.

Consistent with the general lack of sex education available to people with disabilities, there is also an equal lack of knowledge regarding specific reproductive technology and birth control options. The sensitive clinician provides pertinent and accurate education and information (Neistadt \& Freda, 1987). Such a clinician increases sexual knowledge, personal skills, and self-confidence regarding sexual health.

\section{Sexually Transmitted Diseases}

An important consideration in adolescent and adult sexual experiences in the threat to health and fertility from acquiring sexually transmitted diseases 
(STDs). Accurate and explicit information regarding the prevention of STDs, including AIDS, must be available to all children and adults in our society.

Of particular concern are persons with disabilities, who may lack basic sex information and education that makes them more vulnerable for acquiring these diseases. Specific recommendations for STDs and pregnancy prevention must include the use of latex dental dams and of condoms that contain nonoxynol-9. Information about the identification of symptoms and management of various STDs should be as available to individuals with disabilities as it is to others. This requires deliberate efforts by health practitioners.

\section{Options}

Just as artificiality and awkwardness are recognized by nondisabled individuals as an occasional accompaniment to sexual activities, they are also recognized by persons with disabilities. The difference is more one of quantity than quality. However, technology can be helpful for all people. A sizable portion of the nondisabled population has found that sexual diversity and adaptation can be welcome in the bedroom. So, too, people with disabilities can find that technology can be of assistance in sexual activities as it is with other activities of daily living. Surgery has contributed to the advances in technology affecting sexuality (e.g., breast enhancement, penile prosthesis). The rehabilitation practitioner or other professional should supplement the technology with information to facilitate its use. Insights into sexual kinesiology or "the athletics of sex" can be as helpful as the technology itself. Thus, the practitioner assists the person with a disability in coping with pain, fatigue, or spasticity as they affect sexual activity.

Ten percent of the population in the United States has a homosexual orientation (Kinsey \& Pomeroy, 1948; Reinisch, 1990). This should be kept in mind, so that the information and technology provided does not burden the recipient with the need for "gender translation." All medical care should be extended nonjudgmentally (Moses \& Hawkins, 1982).

Physical disabilities that produce sexual dysfunctions or concerns are seen in everyday medical practice. Most practitioners encounter a patient every day with neurotrauma, skeletal disease, cardiopulmonary disabilities, metabolic diseases, dermatologic disorders, pain, malignancy, or sensory disturbances, any one of which can and usually does affect sexuality in major or minor ways.

\section{SPECIFIC REPRODUCTIVE ISSUES AND OPTIONS}

Basic reproductive concerns for men and women in the general population are also of concern for the person with a disability and, in addition, specific topics require particular attention. 


\section{Family Planning}

Medical advice may facilitate pregnancy and the activities of preparing for pregnancy. Spontaneity of intimacy must also be considered. A woman who is beginning intimacy with a disabled man may wish to consider the gender role into which she has been socialized. She may be well advised to be assertive and playful and to take greater initiative. If her partner is paralyzed and cannot initiate physical activities, it may no longer be sufficient for her simply to "not say no."

In the area of family planning, there must be more linkages between those who provide these services and the rehabilitation professionals who understand and work with the problems imposed by physical disabilities. So, too, medical information needs to be tailored to a more diverse population that includes people with physical disabilities. Research in this area is not abundant. Information for the public is not generally available, and many practitioners are unprepared to think about issues of fertility, conception, pregnancy, delivery, or even the monthly occurrence of menstruation for women with physical disabilities (Miller \& Morgan, 1980; Neistadt \& Freda, 1987).

In discussing reproductive options and potentials with an individual with a disability, one must assess whether the disability influences fertility and fertility options (Neistadt \& Freda, 1987). Evaluation must include whether genital sensation and genital function are affected. An example would be a male with a spinal cord injury who is unable to ejaculate. This is a case in which a urologist, specially trained in electroejaculation procedures, would be instrumental in assisting with the evacuation and obtaining of sperm for insemination.

Some birth control methods may be contraindicated for women with specific disabilities. For example, a woman with spinal cord injury who is unable to feel sensation in her genital area and has diminished hand function from quadriplegia would not be a candidate to insert and use a diaphragm. There may also be unrecognized and ongoing medical conditions that affect fertility, such as irregular menses and chronic infection. What reproductive choices might be available for a woman with a disability? She would need to be evaluated to determine if she could physically carry a fetus to full term once she conceived. If this is contraindicated, it may be important to consider other alternatives for reproduction, perhaps using a female surrogate. Evaluating and recommending the type of delivery suited for a woman with a disability is extremely important for the safety of both mother and child. Many obstetricians are not trained to specialize in pregnancies of women with physical disabilities and should consult physiatrists who can assist in consultation and health care of the patient. The presence of sexually transmitted diseases would need to be identified, as would be necessary for any pregnancy. 


\section{Additional Considerations}

There are several other considerations related to the ability to reproduce that are not routinely considered but potentially could have a tremendous impact on an individual with a disability and his or her partner.

People with disabilities frequently live on fixed incomes or are medically subsidized in some way. Therefore, money and finances may be limited or strained, which creates limitations on one's ability to socialize, establish relationships, and find partners (Hanks \& Poplin, 1981). Does the individual with a disability have life skills that include the ability to parent? Does the possibility exist of someone assisting with parenting tasks? In addition, does the individual with the disability require a personal care assistant or someone to assist with general daily living tasks? What is the person's level of independence? What kinds of reasonable accommodations can be provided?

Social skills, body image, self-esteem, and personal integrity are central to one's ability to enjoy life and to participate in the development of relationships, intimate behavior, and preparation for pregnancy, birthing, and parenting. (DeLoach \& Greer, 1981).

When medically evaluating individuals for fertility, the potential for substance abuse, the use or abuse of medications and, as mentioned earlier, a routine evaluation for determination of sexually transmitted diseases or other genital disorders must also be assessed. What previous investigation has been done in the form of tests, procedures, and assessment of sexual activities regarding reproduction? Does the individual understand his or her exact situation and options from a medical perspective? If so, then the individual deserves commendation for determination to learn and challenge a rather passive medical system until there are more answers and solutions.

\section{AGING}

As we age as sexual individuals, we continue to function in our male and female roles, which have been established since adolescence. We experience normal changes of aging, which can include physical disabilities. These changes affect the flexibility and ease with which we are able to conduct our daily lives. Additions such as wheelchairs, new and unfamiliar devices, apparatus, or equipment, which support the body, increase mobility, or enhance and make safe daily living tasks, can be cumbersome and awkward. Changes such as these can be overwhelming to the individual and his or her sense of selfconfidence, dignity, and self-esteem.

We are sexual until our death. However, the gender roles we have carefully created for ourselves can be croded during the aging process by the real- 
ization and personal experience of being slow, being viewed as less important, feeling incapable from time to time, and experiencing a general loss of personal value. These experiences can seriously affect one's confidence and the maintenance of femininity or masculinity. Negative or hesitant reactions from life partners or family members who have become caregivers for the aging individual, health care professionals who do not understand geriatrics, and society in general can increase the vulnerability of one's self-esteem.

The transition from being a healthy adult to one who experiences traumatic or chronic illness or disability can be difficult for many (Trieschmann, 1987). Elderly individuals become keenly aware of their frailties. Inaccurately and inappropriately, society may increase the potential for guilt and shame, which it associates with aspects of aging. This can be directly experienced, for example, by a woman who is incontinent and vulnerable to conspicuous embarrassment in social situations, or by the man who experiences erectile difficulties due to aging or the presence of disease.

It is time that new horizons be recognized and that we cease to add further societal burdens on individuals experiencing natural aging difficulties. Medical and societal recognition of sexual health, sexual development, gender socialization, and fertility issues pertaining to persons with disabilities will serve to pave the way for all individuals to age with sexual dignity.

There has been progressive recognition of the need to liberate people with disabilities who are oppressed. The Rehabilitation Act of 1973 that has been updated and amended is an example of the recognition of the rights of individuals with disabilities. The community of people with disabilities is working together to better state what needs to be addressed by federal legislation. The Americans with Disabilities Act of 1990 (ADA) (PL 101-336) is a civil rights act and an advance for people with disabilities. The ADA does not deal specifically with sexuality, but it does empower the individual.

Sexuality and fertility concerns are natural experiences for persons with disabilities. We cannot artificially separate sexuality from the spectrum of life for anyone.

Civil rights, which are the rallying points of those who are oppressed, include the right to reproductive information that is comprehensive and accurate.

\section{REFERENCES}

1. Allgeier, E.R., \& Allgeier, A.R. (1991). Sexual interactions. Lexington, MA: D.C. Heath.

2. Biklen, D. (1988). The myth of clinical judgment. Journal of Social lssues, 44(1), 127-140.

3. Blackwell-Stratton, M., Breslin, M.L., Mayerson, A.B., \& Bailey, S. (1988). Smashing icons: Disabled women and the disability and women's movements. In M. Fine \& A. Asch (Eds.), Women with disabilities: Essays in psychology, culture, and politics (pp. 306-333). Philadelphia: Temple University Press. 
4. Bogdan, R., Biklen, D., Shapiro, A., \& Spelkoman, D. (1990). The disabled: Media's monster. In M. Nagler (Ed.), Perspectives in disability (pp. 138-143). Palo Alto, CA: Health Markets Research.

5. Brolley, D.Y., \& Anderson, S.C. (1990). Advertising and attitudes. In M. Nagler (Ed.), Perspectives on disability (pp. 147-15I). Palo Alto, CA: Health Markets Research.

6. Cole, S.S. (1986). Facing the challenges of sexual abuse in persons with disabilities. Sexuality and Disability 7(3/4), 71-89.

7. Cole, T.M., \& Cole, S.S. (1990). Rehabilitation of problems of sexuality in physical disability. In F.J. Kottke, G.K. Stillwell, \& J.F. Lehmann (Eds.), Krusen's handbook of physical medicine and rehabilitation (pp. 988-1008). Philadelphia: W.B. Saunders.

8. Darling, R.B. (1988). Parental entrepreneurship: A consumerist response to professional dominance. Journal of Social Issues, 44(1), 141-158.

9. Deloach, C., \& Greer, B.G. (1981). The third disabling myth: The asexuality of the disabled. In Adjustment to severe physical disability: A metamorphosis (pp. 65-99). New York: McGraw-Hill.

10. Ferreyra, S., \& Hughes, K. (1982). Table manners: A guide to the pelvic examination for disabled women and health care providers. Oakland, CA: Sex Education for Disabled People.

11. Fine, M., \& Asch, A. (1985). Disabled women: Sexism without the pedestal. In M.J. Deegan, \& N.A. Brooks (Eds.), Women and disability: The double handicap (pp. 6-23). New Brunswick, NJ: Transaction Books.

12. Finklehor, D. (1979). Sexually victimized children. New York: Free Press.

13. Frank, G. (1988). Beyond stigma: Visibility and self-empowerment of persons with congenital limb deficiencies. Journal of Social Issues, 44(1), 95-117.

14. Goffman, E. (1963). Stigma: Notes on the management of spoiled identity. Englewood Cliffs, NJ: Prentice-Hall.

15. Gordon, S. (1975). Living fully: A guide for young people with a handicap, their parents, their teachers, and professionals. New York: John Day.

16. Gordon, S., \& Gordon, J. (1983). Raising a child conservatively in a sexually permissive world. New York: Simon and Schuster.

17. Griffith, E.R., Cole, S.S., \& Cole, T.M. (1990). Sexuality and sexual dysfunction. In M. Rosenthal, E.R. Griffith, M.R. Bond, \& J.D. Miller (Eds.), Rehabilitation of the adult and child with traumatic brain injury (pp. 206-225). Philadelphia: F.A. Davis.

18. Hahn, H. (1981). The social component of sexuality and disability: Some problems and proposals. Sexuality and Disability, 4(4), 220-234.

19. Hanks, M. \& Poplin, D. (1981). The sociology of physical disability: A review of literature and some conceptual perspectives. Deviant Behavior: An Interdisciplinary Journal, 2, 309328.

20. Kinsey, A.C., Pomeroy, W.B., \& Martin, C.E. (1948). Sexual behavior in the human male. Philadelphia: W.B. Saunders.

21. Miller, S., \& Morgan, M. (1980). Marriage matters: For people with disabilities too. Sexuality and Disability, 3(3), 203-212.

22. Money, J. (1986). Lovemaps: Clinical concepts of sexual/erotic health and pathology, paraphilia, and gender transposition in childhood, adolescence, and maturity. New York: Irvington Publishers.

23. Mooney, T.O., Cole, T.M., \& Chilgren, R.A. (1975). Sexual options for paraplegics and quadriplegics. Boston: Little, Brown.

24. Moses, A.E., \& Hawkins, R.O., Jr. (1982). Counseling lesbian women and gay men: A lifeissues approach. St. Louis: C.V. Mosby.

25. Mullan, P.B., \& Cole, S.S. (1991). Health care providers' perceptions of the vulnerability of persons with disabilities: Sociological frameworks and empirical analyses. Sexuality and Disability, $9(3), 221-243$.

26. Neistadt, M.E., \& Freda, M. (1987). Choices: A guide to sex counseling with physically disabled adults. Malabar, FL: Robert E. Krieger Publishing Co.

27. Rabin, B.J. (1980). The sensuous wheeler: Sexual adjustment for the spinal cord injured. San Francisco: Multi Media Resource Center. 
28. Reinisch, J.M. (1990). The Kinsey Institute new report on sex: What you must know to be sexually literate. New York: St. Martin's Report.

29. Robinault, I.P. (1978). Sex, society, and the disabled. New York: Harper \& Row.

30. Romeis, J.C. (1990). Alienation as a consequence of disability: Contradictory evidence and its interpretations. In M. Nagler (Ed.), Perspectives on disability (pp. 47-57). Palo Alto, CA: Health Markets Research.

31. Rousso, H. (1988). Daughters with disabilities: Defective women or minority women? In M. Fine \& A. Asch (Eds.), Women with disabilities: Essays in psychology, culture, and politics (pp. 139-172). Philadelphia: Temple University Press.

32. Ruffner, R.H. (1984). The invisible issue: Disability in the media. Rehabilitation Digest, $15(4)$.

33. Sandowski, C.L. (1989). Sexual concerns when illness or disability strikes. Springfield: Charles C Thomas.

34. Sha'ked, A. (Ed.). (1981). Human sexuality and rehabilitation medicine: Sexual functioning following spinal cord injury. Baltimore: Williams \& Wilkins.

35. Shapiro, J. (1983). Family reactions and coping strategies in response to the physically ill or handicapped child: A review. Social Science Medicine, 17(14), 913-931.

36. Sobsey, D., \& Varnhagen, C. (1988). Sexual abuse, assault, and exploitation of people with disabilities. Ottawa, Ontario: Health and Welfare Canada.

37. Trieschmann, R.B. (1987). Aging with a disability. New York: Demos Publications. 